\title{
A SINGLE CASE REPORT ON HYPONATREMIA SEIZURE INDUCED BY ACETYLCHOLINESTERASE INHIBITORS
}

\author{
J. SHAREEF ${ }^{*}$, J. JOSEPH ${ }^{1}$, ADITHI K. ${ }^{2}$ \\ 1Department of Pharmacy Practice, NGSM Institute of Pharmaceutical Sciences, Mangaluru, Karnataka, India, ${ }^{2}$ Department of General \\ Medicine, K S Hegde Hospital, Mangaluru, Karnataka, India \\ Email: javedh.shareef@gmail.com \\ Received: 10 Apr 2017 Revised and Accepted: 19 May 2017
}

\begin{abstract}
Donepezil and memantine are the acetylcholinesterase inhibitors most commonly used in clinical practice for the management of mild-moderate dementia in Alzheimer's disease. Electrolyte abnormalities associated with the drugs are commonly encountered in daily clinical practice and often can lead to seizures. Hyponatremia is an uncommon adverse effects of acetylcholinesterase inhibitors. Here we present a case on hyponatremia seizure due to the syndrome of inappropriate antidiuretic hormone (SIADH) caused by the acetylcholinesterase inhibitor donepezil and memantine as other likely causes were ruled out by appropriate investigations. The causality assessment needed to be done according to Naranjo algorithm was found to be 'probable' and the level of severity according to the Hartwig scale was 'Level 5'.
\end{abstract}

Keywords: Adverse drug reactions, Acetylcholinesterase inhibitors, Naranjo Algorithm, Severity level assessment

(C) 2017 The Authors. Published by Innovare Academic Sciences Pvt Ltd. This is an open access article under the CC BY license (http://creativecommons.org/licenses/by/4.0/) DOI: http://dx.doi.org/10.22159/ijpps.2017v9i7.19057

\section{INTRODUCTION}

Dementia is defined as a pathologic deterioration in cognitive abilities that impairs the productive performance of activities of daily living. It refers to a collection of symptoms that occur when brain cells stop working properly severe enough to interfere with occupational or social performance. Episodic memory, the ability to recall events specific in time and place, is the perceptive function most commonly affected. In addition to that, dementia may erode further mental faculties, including language skills, understanding information, spatial skills, judgment and attention [1]

Anticholinesterase inhibitors are the cognitive enhancing medications for treating mild to moderate dementia of Alzheimer's disease and inhibits the cholinesterase enzyme that brings down acetylcholine, a neurotransmitter [2]. However, in general, untoward adverse reactions to drugs can lead to failure in achieving better therapeutic outcomes, prolongs hospital stay and increased healthcare expenditure. Understanding the nature and early identification of adverse drug reactions with appropriate management may help to avoid the severity/complications. Here we present a rare case of seizure secondary to hyponatremia probably induced by donepezil/memantine combination.

\section{CASE REPORT}

A 50 year** old male with a known case of alcoholic dependence syndrome with mental and behavioral disorder and unspecified psychosis and pulmonary tuberculosis (8years back, took antitubercular therapy for $6 \mathrm{mo}$ ) treated with thiamine supplementation and antipsychotics (risperidone $4 \mathrm{mg} 0-0-1$ ) five months back was readmitted to our hospital with chief complaints of walking in sleep, wandering away and reduced sleep for the past three weeks. The patient was assessed clinically and diagnosed with mental and behavioural disorder owing to use of alcohol dependence syndrome (currently abstinent but in a protected environment). The patient continued to have the same treatment during the hospital stay. His vital signs and physical examination results were within normal limits. The laboratory findings of electrolytes revealed the following: sodium- $137 \mathrm{mmol} / \mathrm{l}$, potassium$5.04 \mathrm{mmol} / \mathrm{l}$, chloride- $97.8 \mathrm{mmol} / \mathrm{l}$. Renal and liver function tests were done and found to be within normal limits. However, in view of this persistent symptom such as getting irritated on probing or asking questions, not remembering things told in the previous day and walks away when started questioning, a magnetic resonance image (MRI) scan was done. The report showed a very small right frontal subdural collection along with small vessel ischemic changes and age-related cerebral atrophy. Patient drug therapy was changed and started on acetylcholinesterase inhibitors (a combination of donepezil with memantine $5 \mathrm{mg} / 5 \mathrm{mg} \mathrm{1-0-0)}$ in view of suspected dementia. After three days of starting the drug therapy, the patient developed sudden jerky movements, a worsening of sensorium, behavioural abnormalities, agitation and breathlessness. The patient had an episode of generalized tonic-clonic seizure with tongue bite lasting for about 3-5 min followed by a drop in saturation. He was then shifted to high dependency unit and endotracheal intubation was done for maintenance of oxygenation. Repeated laboratory electrolytes showed sodium-112 $\mathrm{mmol} / \mathrm{l}$ and potassium-4.35 $\mathrm{mmol} / \mathrm{l}$. There was not any history of vomiting, diarrhea or increased thirst. A repeat computed tomography was done which did not show any increase in the subdural hematoma and nor fresh bleed. A lumbar puncture was done which showed a normal study. In view of the temporal relationship between the drugs and the occurrence of events, it was diagnosed to have donepezil-memantine precipitated hyponatremia causing seizures and the drug was stopped. The patient was treated symptomatically with injection Haloperidol ( $5 \mathrm{mg}$ ) and injection lorazepam $(2 \mathrm{mg})$ for agitation and control of seizures. Intravenous fluids with hypertonic saline $3 \%$ $\mathrm{NaCl}$ ) and dextrose with normal saline (DNS) was also provided and improvement was noticed over a period of 4-5 d during which patient was weaned off the mechanical ventilation and shifted to the wards. The patient was restarted with antipsychotics (risperidone o.5 mg 0-0-1) and was discharged with the serum sodium level 136 $\mathrm{mmol} / \mathrm{l}$.

\section{DISCUSSION}

Adverse drug reactions are recognized as the hazards of drug therapy for any class of drugs leading to increased morbidity and mortality. It is expected to increase the length of hospital stay and health care expenditure. Incidence of adverse drug reactions are higher in elderly patients receiving polypharmacy for multiple ailments with multiple comorbid conditions [3-4].

Donepezil and memantine are acetylcholinesterase inhibitors for treating mild to moderate dementia of Alzheimer's disease. It acts reversibly and noncompetitively inhibits centrally-active acetylcholinesterase, the enzyme responsible for hydrolysis of acetylcholine. 
The most common adverse drug reactions associated with acetylcholinesterase inhibitors are insomnia, vomiting, diarrhoea, hypotension, headache, depression, muscle cramps, irritability, restlessness etc [5-7]. There have been voluntary reports of adverse events reported as infrequent events associated with nutritional and metabolic disorders such as hyponatremia during post approval use of acetylcholinesterase inhibitors. Hyponatremia is defined as serum sodium levels of less than $135 \mathrm{mmol} / \mathrm{l}$ and considered severe when the serum level falls below $125 \mathrm{mmol} / \mathrm{l}$. Clinically hyponatremia may be "silent" or may present with malaise, muscle ache, nausea, vomiting or can occur with severe manifestations such as seizures, delirium, coma and even death. Syndrome of inappropriate antidiuretic hormone (SIADH) is the most common cause of hyponatremia in almost one-third of all cases [8-9]. Drugs such as selective serotonin reuptake inhibitors, tricyclic antidepressants, monoamine oxidase inhibitors, diuretics and antiepileptic drugs are known to cause hyponatremia, mostly by inducing the syndrome of inappropriate antidiuretic hormone (SIADH) [10-11]. The mechanism by which acetylcholinesterase inhibitors cause a syndrome of inappropriate antidiuretic hormone (SIADH) remains unclear. In this case, clinical history and investigations were in line with the diagnosis of syndrome of inappropriate antidiuretic hormone (SIADH). Lack of history of polydipsia and onset of hyponatremia with seizures shortly after starting of donepezil and memantine combination and rapid correction of sodium after the withdrawal of the drug makes a relationship with the drug probable.

Causality assessment and the severity level of assessment of the adverse drug reaction were done by using the Naranjo causality scale and the Hartwig's severity assessment scale to assess the causal relationship and level of severity of donepezil and memantine causing hyponatremia. The causality done as per the Naranjo algorithm revealed a "probable" association (Naranjo score 6) between the adverse drug reaction and hyponatremia. The severity assessment scale revealed an adverse drug reaction to be of 'severe' (level 5), suggesting that the suspected drug be withheld, discontinued, and otherwise changed and/or an antidote or other treatment was required. There was a considerable increase in the length of stay and intensive medical care treatment was provided to the patient.

\section{CONCLUSION}

This case study reveals that acetylcholinesterase inhibitors could be causally related to the hyponatremia event. Though there exist inadequate data for the supportive evidence and the precise mechanism is unclear, hyponatremia with clinical history and investigations favors the diagnosis of donepezil with memantine induced syndrome of inappropriate antidiuretic hormone (SIADH) since other causes of hyponatremia were ruled out by suitable investigations. Therefore we suggest a regular monitoring of serum sodium level for all the patients on acetylcholinesterase inhibitors for dementia of Alzheimer's disease to be made a routine clinical practice. So this case enlightens the need for physicians to be aware of the infrequent, potentially life-threatening but preventable hyponatremia as an adverse effect of acetylcholinesterase inhibitors.

\section{ACKNOWLEDGEMENT}

We thank all those colleagues from the department of general medicine for their support and help in writing this case report.

\section{AUTHOR CONTRIBUTION}

We certify that all have participated and contributed sufficiently for the completion of the manuscript and have agreed to have our name listed as a contributor. Neither the content of this manuscript nor any other unified content with substantially similar or comparable substance under our creation or authorship has been published constantly considered for publication elsewhere.

\section{CONFLICT OF INTERESTS}

Declared none

\section{REFERENCES}

1. Robinson L, Tang E, Taylor JP. Dementia: timely diagnosis and early intervention. Br Med J 2015;350:h3029. Doi:10.1136/ bmj.h3029.

2. Brady R, Weinman J. Adherence to cholinesterase inhibitors in Alzheimer's disease: a review. Dementia Geriatr Cognit Disord 2013;35:351-63.

3. Lavan $\mathrm{AH}$, Gallagher $\mathrm{P}$. Predicting risk of adverse drug reactions in older adults. Ther Adv Drug Saf 2016;7:11-22.

4. Brahma DK, Wahlang JB, Marak MD, Sangma MC. Adverse drug reactions in the elderly. J Pharmacol Pharmacother 2013;4:91-4.

5. Colovic MB, Krstic DZ, Lazarevic-Pasti TD, Bondzic AM, Vasic VM. Acetylcholinesterase inhibitors: pharmacology and toxicology. Curr Neuropharmacol 2013;11:315-35.

6. Howard R, McShane R, Lindesay J, Ritchie C, Baldwin A, Barber $\mathrm{R}$, et al. Donepezil and Memantine for moderate-to-severe Alzheimer's disease. N Engl J Med 2012;366:893-903.

7. Ekinci F, Soyaltin UE, Ugur MC, Develi A, Akar H. A rare case of Rhabdomyolysis probably due to donepezil. J Clin Case Rep 2014;4:465.

8. Gross P. Clinical management of SIADH. Ther Adv Endocrinol Metab 2012;3:61-73.

9. Ranga GS, Tomar LR, Narang S, Tripathi P, Jirwal OP. Risperidone causing hyponatremia. J Acute Med 2014;4:133-4.

10. Agrawal P, Bansal M, Upadhyay S, Gupta A. Rifaximin induced hyponatremia: a case report. Asian J Pharm Clin Res 2016;9:22-6.

11. Priya SS, Britto DL, Saravanan T. Zolpidem-induced hyponatremia: a case report. J Clin Diagn Res 2014;8:HD03HD04.

\section{How to cite this article}

- J Shareef, J Joseph, Adithi K. A single case report on hyponatremia seizure induced by acetylcholinesterase inhibitors. Int J Pharm Pharm Sci 2017;9(7):165-166. 